\title{
Frequência de injeções intramusculares na região ventroglútea antes e após oficina de capacitação
}

\author{
Frequency of intramuscular ventrogluteal injections before and after a training workshop
}

Frecuencia de inyecciones intramusculares en la región ventroglútea antes y después de taller de capacitación

Fernanda Raphael Escobar Gimenes ${ }^{1}$, Maristela Priscila Nardo Ramos²

\footnotetext{
1 Enfermeira, Doutora em Enfermagem Fundamental. Professora Doutora da Escola de Enfermagem de Ribeirão Preto da Universidade de São Paulo. Ribeirão Preto, SP, Brasil. E-mail: fregimenes@eerp.usp.br.

${ }^{2}$ Enfermeira. Enfermeira da Clínica Cirúrgica do Hospital Estadual Sumaré. Sumaté, SP, Brasil. E-mail: edi.maristela@hotmail.com.
}

\section{RESUMO}

O objetivo foi identificar a frequência de injeções intramusculares (IMs) na região ventroglútea administradas por profissionais da enfermagem antes e após oficina de capacitação. Do total de 32 sujeitos, participaram do estudo 14 profissionais de um pronto atendimento. Pesquisa exploratória realizada em três fases. A primeira visou identificar a frequência de injeções IMs no ventroglúteo. Na segunda foi realizada oficina de capacitação. A terceira identificou a frequência de injeções IMs no ventroglúteo, após a oficina. Das 525 injeções administradas pela via IM na primeira fase, 520 (99\%) foram na região dorsoglútea e nenhuma na região ventroglútea. Após a oficina, das 448 injeções administradas, 418 (93,3\%) ocorreram na região dorsoglútea e 23 (5,1\%) na região ventroglútea. Apesar do aumento da frequência de injeções no ventroglúteo, o resultado não é significativo. Embora as evidências revelem que a região ventroglútea apresenta menos riscos, os profissionais permanecem priorizando o dorsoglúteo para as injeções.

Descritores: Injeções Intramusculares; Enfermagem; Capacitação Profissional.

\section{ABSTRACT}

The objective was to identify the frequency of ventrogluteal intramuscular injections (IMIs) administered by nursing professionals before and after a training workshop. The participants were 14 out of a total of 32 subjects from an emergency service. This exploratory study had three stages. The first aimed to identify the frequency of ventrogluteal IMIs. The second was the training workshop. The third identified the frequency of ventrogluteal IMIs after the workshop. Of the 525 IMIs in the first phase, 520 (99\%) were in the dorsogluteal site and none in the ventrogluteal site. After the workshop, of the 448 injections administered, 418 (93.3\%) were in the dorsogluteal site and 23 (5.1\%) in the ventrogluteal site. Despite the increased frequency of injections in the ventrogluteal site, the result is insignificant. Although there is evidence that the ventrogluteal site offers lesser risk, professionals continue to prefer administering injections in the dorsogluteal site.

Descriptors: Injections, Intramuscular; Nursing; Professional Training.

\section{RESUMEN}

Se objetivó identificar frecuencia de inyecciones intramusculares (IMs) en región ventroglútea, administradas por profesionales de enfermería antes y después de taller de capacitación. De un total de 32 sujetos, participaron 14 profesionales de una unidad de urgencias. Investigación exploratoria realizada en tres fases. La primera apuntó a identificar frecuencia de inyecciones IMs ventroglúteas. En la segunda se realizó taller de capacitación. La tercera identificó frecuencia de inyecciones INs ventroglúteas luego del taller. De las 525 inyecciones administradas por vía IM en la primera fase, 520 (99\%) fueron en región dorsoglútea y ninguna en región ventroglútea. Luego del taller, de las 448 inyecciones administradas, 418 (93,3\%) fueron en región dorsoglútea y 23 (5,1\%) en región ventroglútea. A pesar del aumento de frecuencia de inyecciones en región ventroglútea, el resultado no es significativo. Aunque las evidencias revelen menos riesgos para región ventroglútea, los profesionales continúan priorizando la región dorsoglútea para inyecciones.

Descriptores: Inyecciones Intramusculares; Enfermería; Capacitación Profesional. 


\section{INTRODUÇÃO}

A via intramuscular (IM) é caracterizada pela introdução de medicamentos dentro do ventre muscular ${ }^{(1)}$. Injeções nesta via são frequentemente administradas nos músculos deltóide, dorsoglúteo (DG), vasto-lateral da coxa e ventroglúteo (VG), e são rapidamente absorvidas. Por esse motivo, a via IM é muito utilizada nos serviços de saúde.

Injeções IMs são frequentes na prática clínica da equipe de enfermagem e requer do profissional, conhecimentos sólidos em disciplinas básicas como anatomia, fisiologia e farmacologia, bem como habilidades técnicas específicas para garantir segurança no procedimento. Este envolve uma complexa série de considerações e de decisões relativas ao volume de solução injetado; ao medicamento a ser ministrado; à técnica e à escolha do local e equipamento; além de considerações sobre a idade dos pacientes, compleição física e condições pré-existentes ${ }^{(2-4)}$. Logo, profissionais qualificados podem minimizar as experiências dolorosas dos pacientes e evitar complicações desnecessárias, favorecendo a segurança e a qualidade do cuidado(3).

Injeções seguras são aquelas que não resultam em danos aos pacientes e que não expõem os profissionais da saúde aos riscos para acidentes biológicos ${ }^{(5)}$, visto que falhas na técnica de preparo e de administração do medicamento podem favorecer os eventos adversos.

Várias complicações estão associadas às injeções IMs ${ }^{(6-7)}$, especialmente às aplicações feitas na região DG. Dentre estas, fibrose da pele e dos tecidos, fibrose muscular e contraturas, paralisias nervosas, abscessos, gangrena, paralisia e hemorragia em pacientes com distúrbios hemorrágicos ${ }^{(8-9)}$.

Estudo realizado no Canadá(8) demonstrou que a complicação mais frequente associada às injeções IMs foi o desconforto do paciente, problema este manifestado por $100 \%$ dos sujeitos que receberam injeção na região do deltóide, por $90 \%$ das pessoas que receberam injeção na região do vasto lateral da coxa, e por $74 \%$ dos indivíduos medicados no DG. Ainda, de acordo com a pesquisa, a lesão de nervos foi a segunda complicação mais citada, tendo esta estado associada a $74 \%$ das injeções administradas na região DG; a 53\% das aplicações feitas na região do deltóide; a $32 \%$ no músculo vasto lateral da coxa, e a 30\% das injeções administradas na região VG.

Reconhece-se que a região VG é particularmente vantajosa por estar livre de estruturas vasculares importantes, por apresentar boa localização, além de ser de fácil acesso e de apresentar tecido subcutâneo menor em relação aos demais sítios de aplicação(10-11). É um local com musculatura bem desenvolvida em todas as idades e deve ser a primeira escolha para as injeções IMs. Porém, tradicionalmente, a região DG é a eleita pelos profissionais de enfermagem na maioria dos $\operatorname{casos}^{(7,12-13)}$.

Pesquisa realizada em um hospital escola do interior

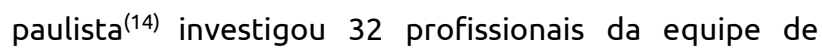
enfermagem quanto às regiões mais utilizadas por eles para a realização de injeções IMs. As regiões eleitas com maior frequência foram a DG $(65,6 \%)$, seguida da deltóidea (31,3\%), face ântero-lateral da coxa $(15,6 \%)$ e VG $(12,5 \%)$.

Evidências sugerem que a região DG não seja mais utilizada para a administração de medicamentos IMS porque expõe os pacientes a riscos desnecessários e inaceitáveis, devendo a região VG ser empregada em todas as faixas etárias(3,10,12). Todavia, a promoção da utilização da região VG para a administração segura de medicamentos injetáveis permanece um desafio, devido, principalmente, à falta de familiaridade dos profissionais com os marcos anatômicos do sítio de aplicação(11,15).

Considerando os saberes presentes na literatura que sugerem a região VG como a mais segura para a administração de injeções IMs, é preciso atualizar os conhecimentos dos profissionais de enfermagem em relação a este procedimento, com vistas a garantir a segurança do paciente e a qualidade da assistência. Рara tanto, faz-se necessário um programa sistematizado de treinamento voltado para a administração segura de medicamentos IMs na região VG.

Estudo prévio(16) visou incentivar os profissionais de enfermagem quanto à utilização da região VG nas injeções IMs, após treinamento por videoconferência. Porém, não foi avaliado o aumento da utilização dessa via na prática profissional. Por conseguinte, o presente estudo foi realizado com o objetivo de identificar a frequência de injeções administradas na região VG, antes e após oficina de capacitação dos profissionais de enfermagem.

\section{MÉTODOS}

Estudo exploratório desenvolvido no Pronto Socorro (PS) do município de Descalvado, interior do estado de São Paulo. Optou-se por realizar o estudo neste município devido às observações feitas pelas pesquisadoras durante 
a supervisão de estágios de alunos de graduação do curso de enfermagem da Universidade Camilo Castelo Branco UNICASTELO, campus Descalvado, onde se constatou o frequente uso da região DG para as injeções IMs por profissionais da enfermagem, ainda que o paciente estivesse manifestando alterações na pele e no músculo decorrentes de injeções sucessivas.

A população foi composta por 32 profissionais de enfermagem atuantes no PS do município. A amostra foi composta por aqueles que aceitaram participar do estudo voluntariamente e por escrito, que tinham mais de 18 anos e que administravam injeções pela via IM, tendo sido excluídos os que, no período da coleta de dados, encontravam-se de férias ou de licença.

A coleta de dados durou cerca de dois anos e foi iniciada no segundo semestre de 2009, após aprovação pelo Comitê de Ética e Pesquisa da Unicastelo (CEP) (Processo $N^{\circ}$ 2654-2874/09) e pela Secretaria Municipal de Saúde. O estudo foi dividido em três fases:

\section{$1^{\text {a }}$ Fase - Identificação da frequência de administração} de medicamentos pela via IM na região ventroglútea, antes da oficina de capacitação

Os dados desta fase foram coletados no segundo semestre de 2009, no PS da Santa Casa de Misericórdia de Descalvado. Para tanto, foi utilizado um instrumento estruturado, desenvolvido pelas próprias pesquisadoras, o qual continha dados do profissional responsável pela injeção IM, faixa etária do paciente atendido, região muscular eleita para a aplicação e o motivo da escolha do local da injeção.

Os dados foram coletados por meio da observação direta e não participante das injeções IMs administradas pelos profissionais da enfermagem atuantes no PS. Foi estabelecida a presença de uma observadora por horário, ou seja, nos períodos matutino e vespertino.

Em seguida, as pesquisadoras tiveram acesso aos prontuários de atendimento médico dos pacientes onde constavam informações sobre o medicamento, tais como nome, dose e via de administração, e dados do paciente atendido, como idade, as quais foram transcritas para o instrumento para posterior análise da frequência de injeções IMs na região VG, antes da oficina de capacitação. $2^{a}$ Fase - Oficina de capacitação para profissionais da enfermagem: administração de medicamentos pela via intramuscular na região ventroglútea

A oficina de capacitação teve carga horária de cinco horas e duração de 50 minutos hora-aula. As aulas foram realizadas no primeiro semestre de 2010, no período da tarde, na sala de reuniões da Santa Casa de Misericórdia de Descalvado, após autorização da enfermeira responsável técnica da instituição. As datas e os horários para a realização da oficina de capacitação foram agendados segundo a disponibilidade dos participantes e em dias consecutivos, com o objetivo de possibilitar a participação dos profissionais de ambos os plantões (par e ímpar).

As aulas teóricas contaram com o apoio de recurso audiovisual (Data Show) e seu conteúdo foi apresentado com o auxílio do programa Power Point.

Рага as aulas práticas de administração de medicamentos IMs na região VG foram utilizadas seringas, agulhas, álcool, algodão, bandeja, luvas, e o manequim de procedimentos de enfermagem para a simulação da técnica.

Nas duas primeiras horas da oficina, as pesquisadoras fizeram uma explanação sobre a administração de medicamentos pela via IM onde foram abordadas as justificativas para a realização da pesquisa, os aspectos éticos, legais e históricos relacionados à técnica, bem como as vantagens e desvantagens do procedimento, critérios para a escolha da região e as características de cada músculo (deltóide, vasto lateral, DG e VG). O foco, no entanto, foi para a administração de medicamentos IMS na região VG.

Nas duas horas subsequentes foram abordados os aspectos inerentes ao conteúdo prático proposto para a oficina, que foram: o reconhecimento das estruturas ósseas envolvidas na localização do sítio de punção, a desinfecção do sítio de aplicação da injeção, e a realização da técnica de administração de medicamentos na região VG, no manequim de procedimentos de enfermagem cedido pelas pesquisadoras. Para tanto, foi utilizada a descrição da técnica proposta pela literatura de enfermagem ${ }^{(17)}$. Houve intervalo de 20 minutos entre as aulas teórica e prática. 


\section{$3^{a}$ Fase - Identificação da frequência de administração} de medicamentos pela via IM na região ventroglútea, após oficina de capacitação

Com o objetivo de avaliar se houve aumento da frequência de administração de medicamentos pela via IM na região VG, no município de Descalvado após a oficina de capacitação, as pesquisadoras utilizaram o mesmo roteiro de observação e seguiram os mesmos passos da coleta de dados da primeira fase da pesquisa. As observações foram realizadas em um período de até 30 dias.

Os dados foram lançados no programa Microsoft Office Excel 2007 e os resultados apresentados, segundo suas frequências absolutas e relativas, por meio de gráficos e tabelas. Em seguida, procedeu-se a análise estatística descritiva por meio do teste t pareado, ao nível de $5 \%$ de probabilidade.

\section{RESULTADOS}

Na $1^{\text {a }}$ fase da investigação participaram um total de $12(37,5 \%)$ profissionais da equipe de enfermagem dos períodos da manhã e da tarde. Destes, oito $(66,7 \%)$ eram técnicos; três (25\%) eram enfermeiros e um (8,3\%), auxiliar de enfermagem; ainda, sete $(58,3 \%)$ eram do sexo feminino e cinco $(41,7 \%)$ eram do sexo masculino.

Nesta fase foi administrado um total de 525 doses de medicamento pela via IM no PS de Descalvado. Destes, 520 (99\%) foram aplicadas na região DG; quatro (0,8\%), na região do vasto lateral da coxa, e uma (0,2\%) foi administrada na região deltoidea. Não houve nenhuma injeção na região VG.

No que diz respeito à faixa etária dos pacientes que receberam as injeções IMs, 355 (67\%) eram adultos entre 18 e 59 anos; 70 (13\%) eram idosos; 56 (10\%) eram adolescentes entre 12 a 17 anos; 43 (8\%) eram crianças entre sete meses a 11 anos, e um (2\%) era lactente (Gráfico 1).

Gráfico 1: Distribuição da frequência de injeções IMs administradas antes da oficina de capacitação, segundo a faixa etária dos pacientes. Descalvado, 2009.

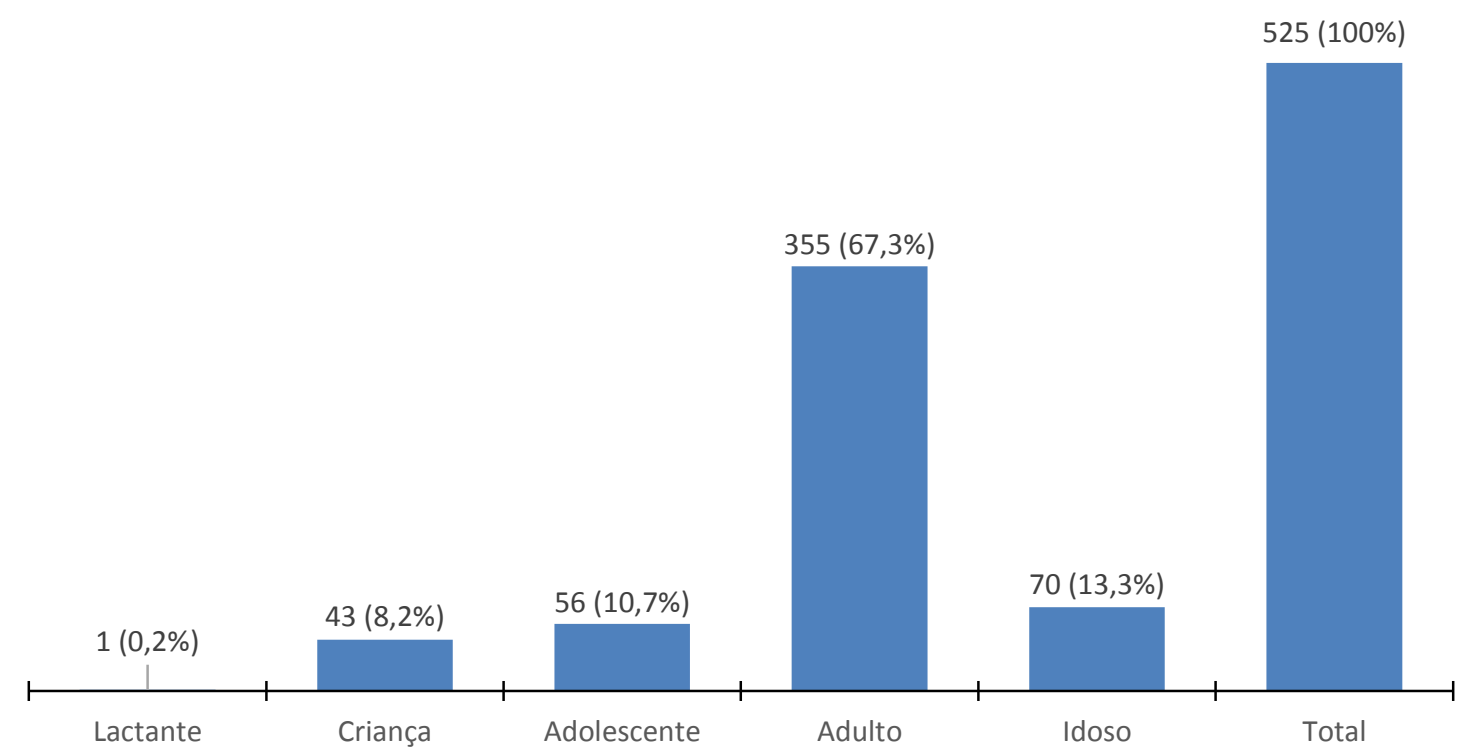

Ainda, a única injeção de vacina foi administrada em lactente no músculo deltoide; crianças com menos de seis meses de vida receberam injeções no DG; e 100\% dos idosos também receberam injeções IMs no DG.

Quando os profissionais da enfermagem foram questionados sobre os motivos que os levaram a optar pela região DG em detrimento de outra, encontramos os seguintes resultados: nas $143(27,2 \%)$ injeções IMS administradas, os profissionais referiram facilidade de acesso; em 141 (26,8\%), referiram mais segurança; em 133 (25,3\%) injeções, os profissionais mencionaram o tipo de medicamento como o mais adequado para o músculo; em 89 (16,9\%) aplicações, a idade do paciente foi o fator determinante; e, em 19 (3,6\%) injeções, os profissionais mencionaram a não aceitação do paciente em relação à outra região (Gráfico 2). 
Gráfico 2: Distribuição da frequência de injeções IMs administradas na região dorsoglútea antes da oficina de capacitação, segundo os motivos da escolha. Descalvado, 2009.

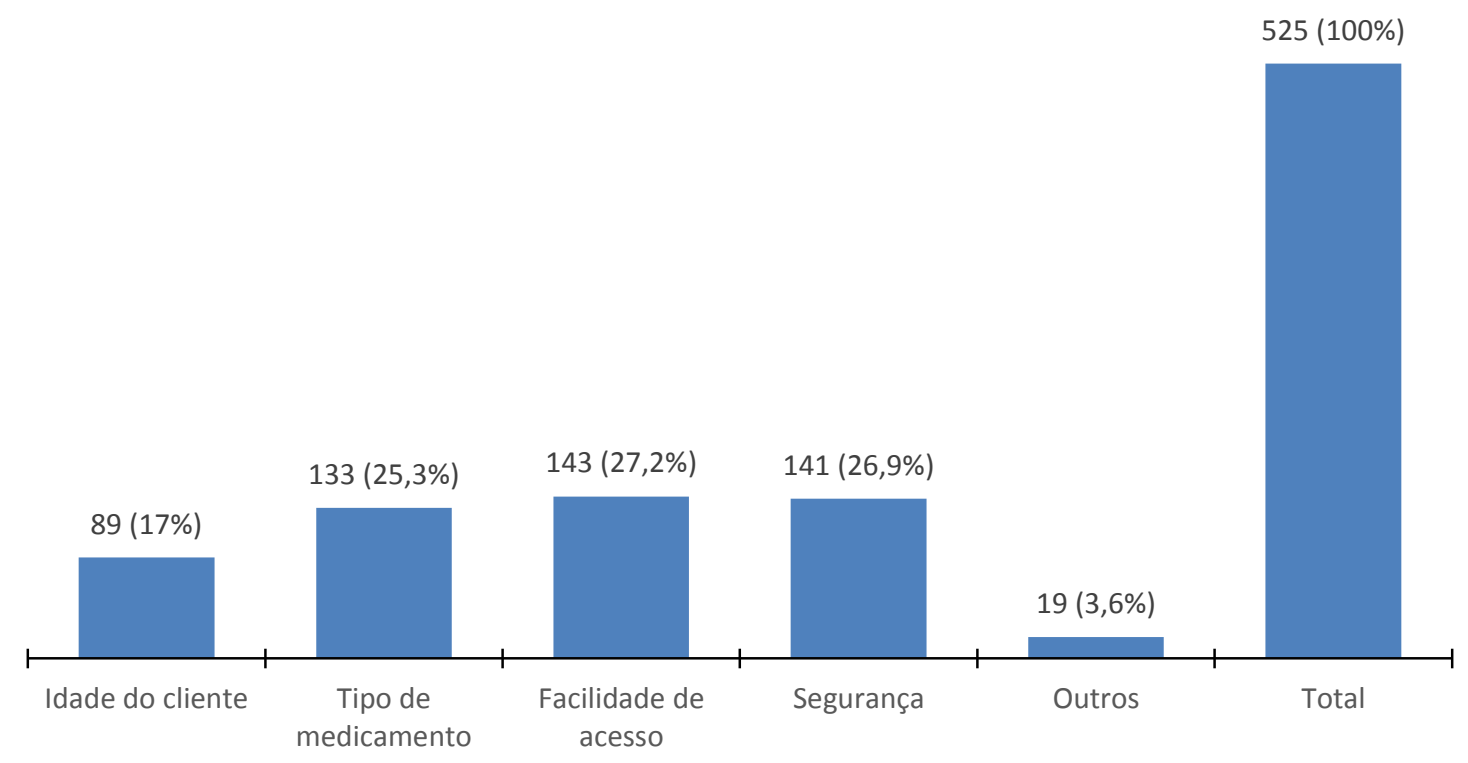

Na segunda fase da pesquisa, participaram da oficina de capacitação 14 (43,8\%) profissionais de enfermagem, do total de 32 . Destes, 11 (78,6\%) eram técnicos, dois $(14,3 \%)$ eram enfermeiros e um $(7,1 \%)$, auxiliar de enfermagem. Todos os profissionais trabalhavam em regime de 12 horas por 36 horas. Durante a realização da oficina, os profissionais de enfermagem demonstraram mais interesse pelo conteúdo prático desenvolvido durante a aula, onde simularam a administração de medicamentos IMs na região VG no manequim de procedimentos.
Já na terceira fase da investigação, participaram 14 $(43,8 \%)$ profissionais da equipe de enfermagem, sendo que 10 (71,4\%) eram técnicos; três $(21,4 \%)$ eram enfermeiros e um $(7,1 \%)$, auxiliar de enfermagem.

Nesta fase foi observado um total de 448 injeções pela via IM no PS de Descalvado. Destas, 418 (93,3\%) foram administradas na região DG; 23 (5,1\%) na região VG; quatro $(0,9 \%)$ na face anterolateral da coxa e três $(0,7 \%)$ no deltoide (Tabela 1$)$.

Tabela 1: Distribuição das injeções IMs administradas no PS, segundo a região muscular, antes e após a oficina de capacitação. Descalvado, 2010

\begin{tabular}{|c|c|c|c|c|}
\hline \multirow{3}{*}{ Regiões } & \multicolumn{4}{|c|}{ Frequência } \\
\hline & \multicolumn{2}{|c|}{$1^{\circ}$ Fase } & \multicolumn{2}{|c|}{$2^{\circ}$ Fase } \\
\hline & $\mathbf{n}$ & $\%$ & $\mathbf{n}$ & $\%$ \\
\hline Deltóide & 1 & 0,2 & 3 & 0,7 \\
\hline Vasto-lateral & 4 & 0,8 & 4 & 0,9 \\
\hline Dorsoglúteo & 520 & 99 & 418 & 93,3 \\
\hline Ventroglúteo & 0 & 0 & 23 & 5,1 \\
\hline Total & 525 & 100 & 448 & 100 \\
\hline
\end{tabular}

Os motivos que levaram os profissionais a utilizarem a região VG na terceira fase do estudo foram, o estado nutricional do paciente, referido em duas $(8,7 \%)$ situações; facilidade de acesso ao local em sete $(30,4 \%)$ situações; e aceitação do paciente em 14 (60,9\%) casos.

No que diz respeito aos motivos que levaram os profissionais de enfermagem a optarem pela região DG, após a oficina de capacitação, obtivemos os seguintes resultados: das 418 injeções administradas, a facilidade de acesso foi mencionada 327 (78,2\%) vezes.

Além da facilidade de acesso, os profissionais apontaram outros motivos para utilização da região DG em detrimento da VG após a oficina de capacitação. Dentre eles, destacaram-se: mais segurança do profissional para realizar a aplicação do medicamento no DG; o costume pela utilização da região; e Rev. Eletr. Enf. [Internet]. 2013 jul/set;15(3):678-86. Disponível em: http://dx.doi.org/10.5216/ree.v15i3.19055. doi: 10.5216/ree.v15i3.19055. 
desconhecimento de outras áreas para administração de volumes superiores a $4 \mathrm{ml}$.

Também foi constado nesta fase que quatro $(0,9 \%)$ injeções foram administradas na região anterolateral da coxa de lactentes. Nestas situações, os profissionais referiram que a idade do paciente foi o fator determinante na escolha. Ainda, três injeções de imunobiológicos foram feitas em crianças entre sete meses e 11 anos na região do deltoide.

Para as injeções DGs realizadas em crianças com menos de seis meses de idade, os profissionais citaram o estado nutricional das crianças como motivo pela escolha do local, as quais foram consideradas magras pelos profissionais, além de perceberem a musculatura pouco desenvolvida na região anterolateral da coxa.

Apesar de termos observado aumento no número de injeções administradas na região VG após a oficina de capacitação, este resultado não pode ser considerado significativo. Após a aplicação do teste $t$, verificou-se que o t observado era menor que o t- crítico $(0,69<2,35)$ indicando diferença não significativa entre os resultados obtidos na primeira e na terceira fase da pesquisa, ao nível de $5 \%$ de probabilidade. Uma confirmação desta não significância é o valor de correlação de Pearson, entre a primeira e a terceira fases, que é de 0,999 (99,9\%) (Tabela 2).

Tabela 2: Resultados do teste t pareado, aplicado à frequência de injeções intramusculares na primeira e na terceira fases da pesquisa. Descalvado, 2010

\begin{tabular}{ccc}
\hline Teste-t: duas amostras em par para médias & $\mathbf{1}^{\circ}$ Fase & $\mathbf{2}^{\circ}$ Fase \\
\hline Média & 131,25 & 112 \\
Variância & 67170,25 & 41700,67 \\
Correlação de Pearson & 0,999 & - \\
Stat t & 0,69 & - \\
$\mathrm{P}(\mathrm{T}<=\mathrm{t})$ uni-caudal & 0,27 & - \\
$\mathrm{t}$ crítico uni-caudal & 2,35 & - \\
$\mathrm{P}(\mathrm{T}<=\mathrm{t})$ bi-caudal & 0,54 & - \\
$\mathrm{t}$ crítico bi-caudal & 3,18 & - \\
\hline
\end{tabular}

\section{DISCUSSÃO}

Na primeira fase da pesquisa foram administradas 525 injeções pela via IM, das quais $99 \%$ foram aplicadas na região DG e nenhuma na região VG. Estes achados são semelhantes àqueles identificados por pesquisadores canadenses, os quais revelaram que o DG foi o músculo eleito pelos profissionais da enfermagem na maioria das injeções, representando $71 \%$, enquanto que a região VG havia sido utilizada em apenas $14 \%$ dos $\operatorname{casos}^{(8)}$.

Pesquisa realizada em um hospital de ensino do interior paulista ${ }^{(14)}$ também revelou que a região mais empregada pela equipe de enfermagem na administração de injeções IMs foi a DG, representando $65 \%$ do total de injeções, seguidas da região deltoide $(31,3 \%)$, vasto lateral da coxa $(15,6 \%)$ e VG $(12,5 \%)$

Percebe-se que em ambos os estudos, a região VG foi utilizada por alguns profissionais, ao contrário do que se verificou nesta pesquisa, pois não houve nenhuma aplicação de injeção na região VG na primeira fase do estudo. Isto pode ser justificado pelo fato de que em hospitais-escola, os profissionais de saúde recebem treinamento continuamente. Além disso, a presença de estudantes de graduação provenientes das universidades ajuda a manter os profissionais atualizados. Contudo, o uso da região DG ainda é muito maior quando comparado à região VG.

Conforme observado no Gráfico 1, crianças com menos de seis meses de idade receberam injeções IMs na região DG. A região recomendada para a administração de injeções nesta faixa etária é a do vasto lateral da coxa, a qual possui massa muscular mais desenvolvida ${ }^{(3)}$. No entanto, pesquisadores afirmam que a região VG deve ser empregada em todas as faixas etárias, incluindo crianças com menos de seis meses de idade ${ }^{(12)}$.

A área é considerada a opção mais segura para as injeções IMs, uma vez que evita a punção acidental de nervos e de vasos sanguíneos, havendo poucos relatos de complicações associadas ${ }^{(3)}$.

Em relação às quatro injeções administradas na região do vasto-lateral da coxa, estas foram feitas em lactentes. O músculo, nesta faixa etária, é mais desenvolvido que o DG, sendo o mais adequado para a administração de medicamentos por via IM nesta idade ${ }^{(3)}$.

No que diz respeito ao lactente que recebeu injeção de vacina no músculo deltoide, esta deveria ter sido feita na região do vasto-lateral da coxa, visto que essa região 
só é indicada para indivíduos acima dos seis anos de idade $^{(12)}$.

Devido às variações anatômicas do nervo axilar, e considerando o potencial de sequela causada pela lesão neural, este músculo não deve ser o sítio de primeira escolha para injeção IM(2-3,5,8,17).

Para as injeções DGs realizadas em crianças com menos de seis meses de vida, os profissionais da enfermagem devem se atentar para os riscos associados a esta prática, visto que o músculo só se desenvolve a partir da deambulação(3), não sendo adequado para a faixa etária.

Também foi verificada a utilização da região DG em todos os idosos na primeira fase da pesquisa. Pesquisadores ${ }^{(3,18)}$ afirmam que a musculatura nesta população é menor e, portanto, mais susceptível a lesões devido às mudanças que ocorrem durante o processo de envelhecimento, devendo a região ser evitada nesta faixa etária. Para os investigadores, a equipe de enfermagem deve optar pela região VG nas injeções IMs.

Há de se advertir que esta área não tem sido mais recomendada para a administração de medicamentos por via $\mathrm{IM}$, em diversas localidades do mundo, devido às complicações associadas ao sítio(3).

Em relação aos motivos que favoreceram a escolha da região DG, em detrimento de outros músculos, verificamos que os profissionais da enfermagem referiram com mais frequência a facilidade de acesso à área e a segurança para realizar as injeções neste músculo.

Alguns profissionais afirmam que a facilidade de acesso ao músculo DG contribui para a dinâmica do serviço na unidade de pronto atendimento, visto que o número de pacientes atendidos é grande. Segundo eles, delimitar a região VG é trabalhoso e é considerado um procedimento demorado.

Estudo conduzido na Nova Zelândia(19) com 173 enfermeiros que trabalhavam em diversas instituições de saúde demonstrou que os profissionais não utilizam a região VG nas injeções IMs devido ao desconhecimento de suas vantagens, às dificuldades para localizar o sítio de aplicação, e à relutância em mudar sua prática. No entanto, a região VG é considerada a mais vantajosa para a administração de medicamentos IMs, além de ser a que oferece menos riscos para o paciente ${ }^{(8,18,20)}$.

Pesquisa realizada com enfermeiros de diversas instituições de saúde no Canadá(8) revelou que a opção pela escolha do músculo varia significativamente com a idade, nível de preparo do profissional, tempo de atuação na área da enfermagem, e conhecimento sobre os riscos de dano ao nervo associados às injeções IMs no DG.

No que diz respeito à segunda fase da pesquisa, as simulações ocorreram repetidas vezes, até que os profissionais fossem capazes de realizá-las de maneira satisfatória, segundo a descrição da técnica prevista na literatura(17). Uma forma eficiente de favorecer o desenvolvimento técnico dos profissionais de enfermagem é a promoção de oportunidades de ensino fundamentadas na conscientização da educação(21-22).

Por meio dos programas de capacitação, de treinamentos contínuos e de cursos estruturados, os processos educativos têm como objetivo o desenvolvimento dos profissionais de enfermagem ${ }^{(22)}$, devendo as instituições de saúde investirem mais nestes programas, em favor da segurança do paciente e da qualidade da assistência de enfermagem.

Na terceira fase da pesquisa, a região DG também foi - local mais utilizado para a administração de medicamentos por via IM, constituindo 93,3\% do total de injeções, mesmo após a realização da oficina de capacitação, conforme Tabela 1.

Uma investigação(18) constatou que injeções administradas na região DG causaram lesões, como fibrose, abscessos, lesão no nervo ciático, necrose de tecido, contração muscular, dor e gangrena, mas que não houve nenhuma complicação associada à injeção IM na região VG, devendo este músculo ser a primeira opção de escolha nas injeções IMs. Ainda, a região VG deve ser empregada em pacientes emagrecidos, edemaciados ou que apresentarem tecido subcutâneo abundante, além de poder ser utilizada com o paciente em qualquer decúbito(6,14).

Tanto na $1^{\text {a }}$ quanto na $3^{a}$ fase do estudo, houve predomínio de injeções IMs na região DG, resultado este semelhante ao encontrado em estudo canadense ${ }^{(8)}$. Notase que, apesar da região VG ter sido introduzida na prática clínica há mais de 50 anos por Hochestteter, ela continua sendo pouco utilizada pelos profissionais da enfermagem.

Este cenário pode estar associado à falta de conhecimento da equipe em relação à delimitação correta da região e ao desconhecimento de suas vantagens e desvantagens, visto que muitos destes profissionais 
afirmaram não ter estudo do assunto durante sua formação profissional.

A baixa frequência de utilização da região VG neste estudo também pode estar relacionada à falta de participação dos enfermeiros na oficina de capacitação. Na qualidade de líderes e de educadores, fica evidente a necessidade dos enfermeiros de se manterem atualizados em relação ao conhecimento voltado para as injeções IMs, de modo a adequar sua prática assistencial e de transmitir estes conhecimentos a todos os membros da equipe de enfermagem, visto que o enfermeiro é visto como fonte de influência e de liderança nas instituições hospitalares ${ }^{(23)}$.

Pesquisa(24) revelou que os eventos adversos associados aos medicamentos estão frequentemente relacionados à falta de experiência e de treinamento dos profissionais que estão na linha de frente do atendimento.

A promoção de programas de educação permanente configura-se como um aspecto importante para que os profissionais de enfermagem adquiram competências técnicas e científicas inerentes à administração de medicamentos pela via IM de maneira segura.

Por conseguinte, as instituições devem se responsabilizar pela atualização permanente de suas equipes, tanto dos novos como dos mais antigos, de modo a favorecer e a manter a qualidade e a segurança no atendimento prestado aos pacientes.

Todavia, de modo geral, os pacientes desconhecem a região VG como a mais segura para a administração de medicamentos por via IM. O desconhecimento da população em relação à região VG contribui para a não adesão do profissional e do paciente ao procedimento. É imprescindivel que a equipe de enfermagem se apodere do conhecimento atualmente disponível para educar os pacientes quanto aos benefícios do uso da região VG nas injeções IMs, em detrimento de outros músculos.

Nota-se que, apesar dos profissionais terem participado da oficina de capacitação, onde foram abordadas questões referentes às contraindicações, às vantagens e às desvantagens dos músculos, à facilidade de acesso à região VG em qualquer decúbito e à possibilidade de utilizá-la em todas as faixas etárias, os profissionais não a incorporaram a sua prática profissional.

Estudos $^{(8-9,15,25)}$ revelam que muitos enfermeiros são resistentes à adoção de novos métodos, e que as evidências científicas não são suficientes para promover mudança em suas práticas. Segundo os pesquisadores, as principais razões para esta relutância incluem a falta de informação adequada para a utilização da região e a incerteza quanto à localização correta do local de aplicação das injeções.

Esta atitude expõe os pacientes sob terapia medicamentosa por via IM a riscos de danos desnecessários, devendo a equipe de enfermagem do município investigado rever suas práticas visto que, atualmente, a enfermagem tem procurado embasar suas ações nas melhores evidências científicas, em favor da segurança dos pacientes e da qualidade da assistência prestada nos serviços de saúde.

\section{CONSIDERAÇÕES FINAIS}

Esperava-se que a região VG fosse utilizada com mais frequência pela equipe de enfermagem, após a oficina de capacitação, o que configuraria uma importante mudança na prática assistencial e um avanço na qualidade da assistência prestada aos pacientes. Porém, os resultados demonstraram uma situação parecida com aquela identificada na primeira fase da pesquisa.

Apurou-se que o principal motivo que contribuiu com a escolha do músculo DG nas injeções, em detrimento do VG, foi a facilidade de acesso ao local, de modo que os profissionais da enfermagem pareceram não considerar a segurança do paciente na execução do procedimento. Verificou-se, ainda, que a região VG é muito pouco utilizada pela equipe, mesmo após eles terem participado da oficina de capacitação.

Apesar das evidências científicas demonstrarem que a região VG é a mais vantajosa e com menos riscos para os eventos adversos, os profissionais permanecem priorizando a região DG para a administração de injeções IMs no dia a dia do trabalho.

Os achados deste estudo revelam o apego dos profissionais de enfermagem às práticas tradicionais, sendo algumas delas obsoletas. A desconsideração da equipe pela segurança do paciente também chama a atenção, devendo a instituição investir em estratégias que favoreçam a mudança no comportamento em favor da segurança e da qualidade da assistência prestada aos pacientes.

Os resultados do estudo apontam, finalmente, que tais aspectos reforçam lacunas importantes que merecem investimento de futuras investigações que possibilitem 
não apenas o treinamento, mas o monitoramento de grupos de profissionais já treinados e o acompanhamento para sanar dúvidas e estimular confiança para que os mesmos utilizem a região VG como opção para as injeções IMs.

\section{REFERÊNCIAS}

1. Meneses AS, Marques IR. A proposal for a geometrical delimitation model for ventro-gluteal injection. Rev Bras Enferm. 2007;60(5):552-8.

2. Agac E, Gunes UY. Effect on pain of changing the needle prior to administering medicine intramuscularly: a randomized controlled trial. J Adv Nurs. 2011;67(3):563-8.

3. Conselho Regional de Enfermagem do Estado de São Paulo. Administração de medicamentos por via intramuscular. São Paulo: COREN-SP; 2010.

4. Hunter J. Intramuscular injection techniques. Nursing Standard. 2008;22(24):6.

5. World Health Organization. WHO best practices for injections and related procedures toolkit. Geneva: World Health Organization; 2010. ix, 55 p. p.

6. Huffaker L. Are dorsogluteal injections dangerous? 30ed: Kentucky Board of Nursing; 2012. 1 p.

7. Cornwall J. Are nursing students safe when choosing gluteal intramuscular injection locations? Australas Med J. 2011;4(6):315-21.

8. Walsh L, Brophy K. Staff nurses' sites of choice for administering intramuscular injections to adult patients in the acute care setting. J Adv Nurs. 2011;67(5):1034-40.

9. Plotkin SA, Orenstein WA, Paul A. Offit MD. Vaccines: Stanley A. Plotkin, Walter A. Orenstein and Paul A. Offit: SAUNDERS W B Company; 2008.

10. Mishra $P$, Stringer MD. Sciatic nerve injury from intramuscular injection: a persistent and global problem. Int J Clin Pract. 2010;64(11):1573-9.

11. Sim G. Treatment injury case study: Sharing information to enhance patient safety. New Zealand: The Accident Compensation Corporation; 2012.

12. Malkin B. Are techniques used for intramuscular injection based on research evidence? Nurs Times. 2008;104(50-51):4851.

13. Pfeifer GM, Potera C. Most nurses don't follow guidelines on im injections. The American Journal of Nursing. 2011;111(8):1. 14. Godoy S, Nogueira MS, Mendes IA. Intramuscular drug administration: analysis of knowledge among nursing professionals. Rev Esc Enferm USP. 2004;38(2):135-42. 15. Cocoman A, Murray J. Recognizing the evidence and changing practice on injection sites. $\mathrm{Br} \mathrm{J}$ Nurs.

2010;19(18):1170-4.

16. Godoy S, Nogueira MS, Hayashida M, Mendes IAC. Administração de injetáveis por via intramuscular na região ventroglútea: avaliação após treinamento por videoconferência. REVRENE. 2003;4(1):6.

17. Potter $P$, Perry A. Fundamentos de enfermagem. 7 ed. Rio de Janeiro: Elsevier; 2009.

18. Carter-Templeton H, McCoy T. Are we on the same page?: a comparison of intramuscular injection explanations in nursing fundamental texts. Medsurg Nurs. 2008;17(4):237-40. 19. Floyd S, Meyer A. Intramuscular injections--what's best practice? Nurs N Z. 2007;13(6):20-2.

20. Gabrielli C, Alves JM, Vargas JC, Saito MM, Lago MM. Bases anatômicas da injeção intramuscular ventroglútea e implicações clínicas. Acta Sci Med. 2009;2(1):8.

\section{AGRADECIMENTOS}

As autoras agradecem às enfermeiras Ariadne Eleonora Trombert Silveira, Lígia Dorlly Trombert Silveira, Fernanda Xavier da Silva e Nayara Aparecida Devitto pela contribuição na coleta e na análise dos dados.

21. Paschoal AS, Mantovani MdF, Méier MJ. Percepção da educação permanente, continuada e em serviço para enfermeiros de um hospital de ensino. Revista da Escola de Enfermagem da USP. 2007;41:478-84.

22. Silva GMd, Seiffert OMLB. Educação continuada em enfermagem: uma proposta metodológica. Revista Brasileira de Enfermagem. 2009;62:362-6.

23. Souza M, Nassar A, Zanon T. Liderança nas rodas de educação permanente: habilidade a ser desenvolvida pelo enfermeiro. Nursing. 2009;12(136):4.

24. Kosnik LK, Brown J, Maund T. Patient safety: learning from the aviation industry. Nurs Manage. 38. United States. 2007; quiz 1:25-30.

25. Cocoman A, Murray J. Intramuscular injections: a review of best practice for mental health nurses. J Psychiatr Ment Health Nurs. 2008;15(5):424-34.

Artigo recebido em 23/06/2012.

Aprovado para publicação em 19/07/2013.

Artigo publicado em 30/09/2013. 